\title{
Left Atrial Compression by a Mediastinal Lymphoma Presenting with Paroxysmal Atrial Fibrillation
}

\author{
Saqib Ahmed MD ${ }^{1}$, Hector Marchand MD ${ }^{1}$, Karen Cedeno-Kelly MD ${ }^{1}$ \\ Spectrum Health, Butterworth Hospital, Grand Rapids, Michigan, USA
}

\begin{abstract}
Extrinsic left atrial compression is an uncommon source of hemodynamic compromise and arrhythmias caused by many mediastinal structures. We describe a case of a 72-year old woman presenting with symptoms of confusion and atrial fibrillation. Transthoracic echocardiography demonstrated extrinsic left atrial compression caused by an enlarged lymph node. Compression of the left atrium caused impaired left atrial filling, leading to pulmonary vascular congestion and pulmonary edema. To our knowledge, the present case is the first reported case of mediastinal follicular lymphoma presenting with atrial fibrillation and heart failure by externally compressing the left atrium.
\end{abstract}

Keywords: LA, Left Atrium

\section{Introduction}

Because of its central position within the thorax, the heart can be encroached upon by masses originating in anterior, posterior, or superior mediastinum. Anterior masses tend to compress the right heart chambers; posterior masses impinge on or compress the left atrium or ventricle, particularly the former. Lymph node enlargement represents a frequent cause of mediastinal masses which potentially can cause compression of thoracic and mediastinal structures.

\section{Case}

This patient is a 72 year old female who has a history of follicular B-cell lymphoma in the retroperitonium and had completed 6 rounds of R-ICE therapy as well as one cycle of R-GemMOX presented to the ED with weakness, fatigue, dyspnea on exertion, confusion, multiple bruises and decrease oral intake for several days. Initial physical examination revealed several bruises on her head as well as her lower extremities bilaterally.

Patient was subsequently admitted to the HematologyOncology service for management of progressive lymphoma. During her stay, patient developed worsening confusion as well as new-onset paroxysmal atrial fibrillation with RVR associated with some respiratory distress. A CXR revealed an enlarging right mediastinal mass, left lower lobe opacity, rightward deviation of the trachea, and new enlargement of heart size. A complete echocardiogram revealed a large external cardiac mass compressing the left atrium as well as decrease in left ventricular ejection fraction to $37 \%$. A CT of the thorax showed several mediastinal masses, largest measuring 8.4 x 5.2 causing significant mass effect upon the left ventricle and rightward and anterior displacement of the thoracic esophagus, along with proximal distension of the esophagus. Scan also showed left lower lobe collapse with a very large pleural effusion as well as moderate to large right pleural effusion with mild right lower lobe compression atelectasis. Pulmonary vasculature was unremarkable. Given patient's history of B-cell lymphoma, preliminary diagnosis was relapse and metastasis of lymphoma, unresponsive to chemotherapy. Her condition was deemed untreatable. She eventually passed away as a cause of acute respiratory failure secondary to this mediastinal mass causing mass effect upon left mainstem bronchus, lower lobe bronchi, left atrium and esophagus.

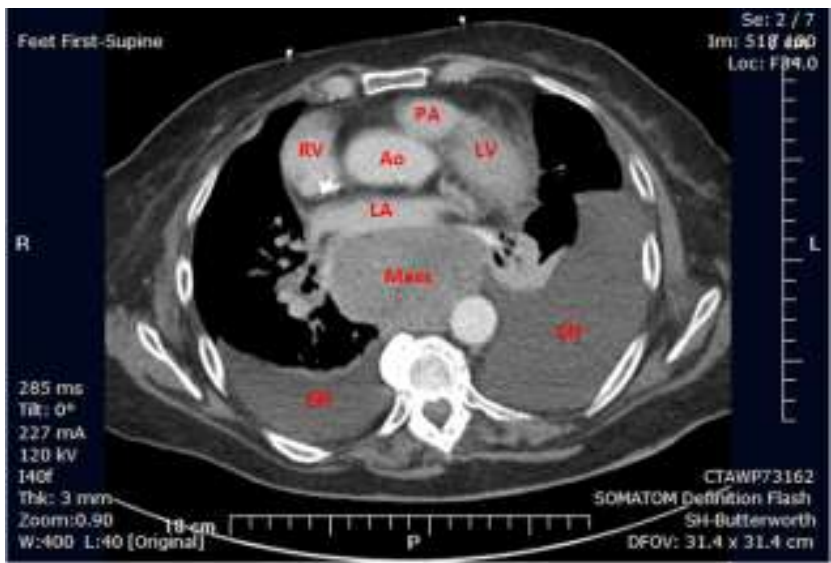

Figure 2: CT Thorax with IV contrast showing mediastinal mass compressing on left atrium. RV: right ventricle; Left ventricle (LV); Left atrium (LA);

Pulmonary artery (PA); Aorta (Ao); Effusion (Eff); Mediastinal lymphoma (Mass)

\section{Discussion}

The left atrial compressing structures are divided into four anatomic groups: (i) gastrointestinal structures, which are the most common, (ii) mediastinal structures, (iii) aorta and intrapericardial structures, and (iv) pulmonary structures. Explanatory examples of left atrial compression with different causes and various levels of severity are presented. D'Cruz et al divided the relation between mediastinal masses and the heart into three categories: proximity (a contiguous or adjacent structure without chamber deformation), encroachment (distortion of normal cardiovascular architecture without hemodynamic effect),

\section{Volume 6 Issue 12, December 2017}




\section{International Journal of Science and Research (IJSR) \\ ISSN (Online): 2319-7064 \\ Index Copernicus Value (2016): 79.57 | Impact Factor (2015): 6.391}

and compression. Clinical manifestations of compression include hypotension, hypoxia, tachypnea, and tachycardia

as in the present patient.

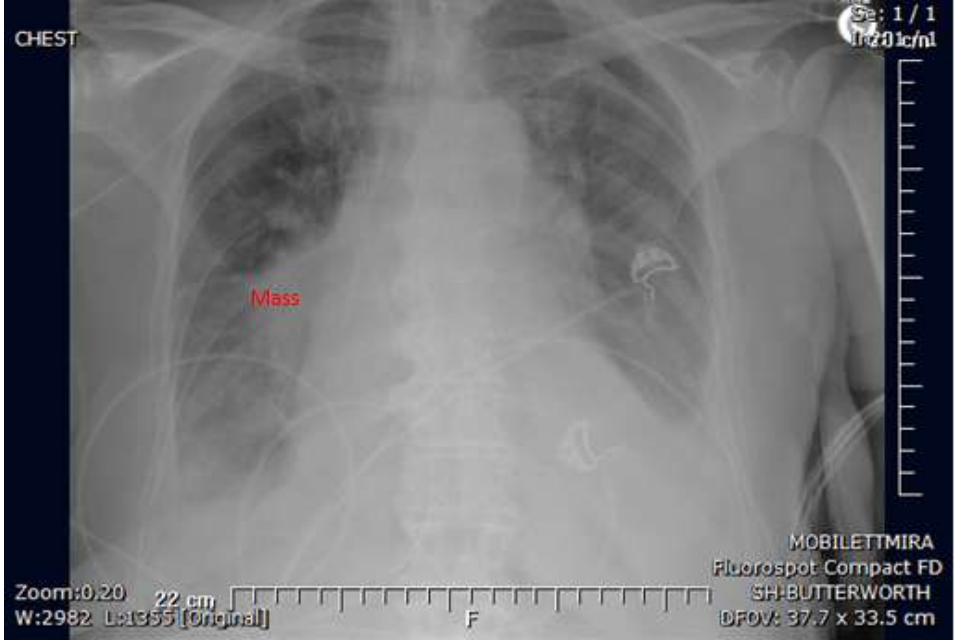

Figure 1: AP Chest Xray showing right mediastinal mass. Bilateral pleural effusions also appreciated

Our patient presented with an unusual cause of atrial fibrillation because of extrinsic compression of the left atrium. Possible mechanisms for the observed decreased cardiac output and pulmonary congestion include obstruction of left atrial inflow and indirect impairment of ventricular filling. Chorro et al also described case with extrinsic compression of the right atrium due to a primary mediastinal germ cell tumor. Transthoracic echocardiography is the test of choice for diagnosing left atrial compression. In contrast to intrinsic atrial abnormalities, such as thrombus or myxoma, extrinsic masses move asynchronously with atria. Transesophagealechocardiography may also be useful for identification of masses adjacent to left atrium.Even a slight impression of the left atrium can be visualized using standard echo views. The round shape of the atrial wall becomes distorted. These characteristics make the use of trans-esophageal echocardiography (TEE) in diagnosing left atrial impression in most situations unnecessary. The other potential important diagnostic tool to disclose the cause of heart failure is thoracic CT. Importantly, thoracic CT might be used not only to determine the interrelation between the mass and cardiac structure but also to identify the etiologic cause of the disease. However, in the consideration of noninvasive and safety properties, transthoracic echocardiography, in our view, should be performed early. In cases of uncertain or doubtful findings additional tools such as thoracic CT should be kept mind. Extrinsic compression of the left atrium is an unusual cause of congestive heart failure. In conclusion; this is a rare and unique cause of atrial fibrillation due to extrinsic compression of the atrium by a metastatic mediastinal lymphoma. Left atrial

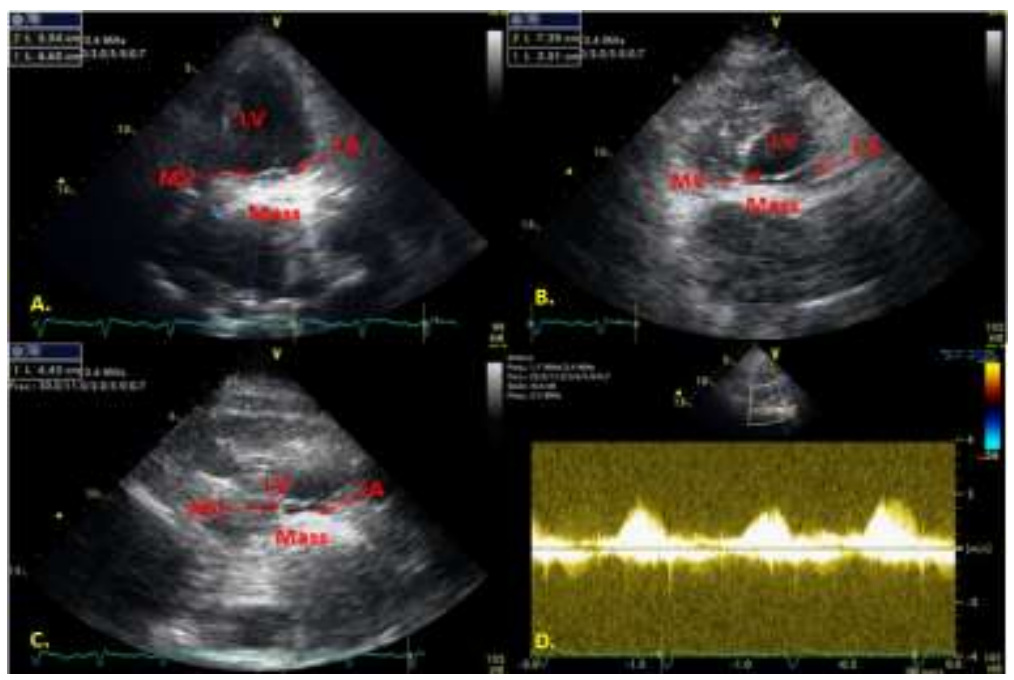

Figure 3: Echocardiogram showing left atrial compression. LA measures $0.56 \mathrm{~cm}$ in a 2-D plane $(\mathrm{N}=2.7-4 \mathrm{~cm})$. Study limited due to patient motion. (A) Apical view (B) Short view (C) Long view (D) Left atrial doppler showing elevated outflow velocity from LA to LV; Max velocity $1.8 \mathrm{~m} / \mathrm{sec}(\mathrm{N}=<2.1 \mathrm{~cm} / \mathrm{sec})$. Left ventricle (LV); Left atrium (LA); Mitral valve (MV); mediastinal mass (Mass)compressionis associated with severe hemodynamic compromiseand a quick and accurate diagnosis is of critical importance.

Volume 6 Issue 12, December 2017 www.ijsr.net 


\section{International Journal of Science and Research (IJSR) \\ ISSN (Online): 2319-7064 \\ Index Copernicus Value (2016): 79.57 | Impact Factor (2015): 6.391}

Mediastinal tumors span a wide histopathological and radiological spectrum. Neurogenic tumors, germ cell neoplasms and foregut cysts represent majority of childhood lesions, whereas primary thymic neoplasms, thyroid masses and lymphomas are the most common in adults.

Primary mediastinal lymphoma usually occurs in the anterior mediastinum.Malignant lymphoma accounts for nearly $20 \%$ of all mediastinum neoplasms in adults and
$50 \%$ in children Hodgkin lymphoma represents approximately $50-70 \%$ while non-Hodgkin lymphoma comprises 15-25\%. The two most common forms of mediastinal non-Hodgkin disease (NHD) include diffuse large B-cell lymphoma and T-cell lymphoblastic lymphoma. Themost common CT appearance is that of a large mediastinal mass compressing the airway and cardiovascular structures.

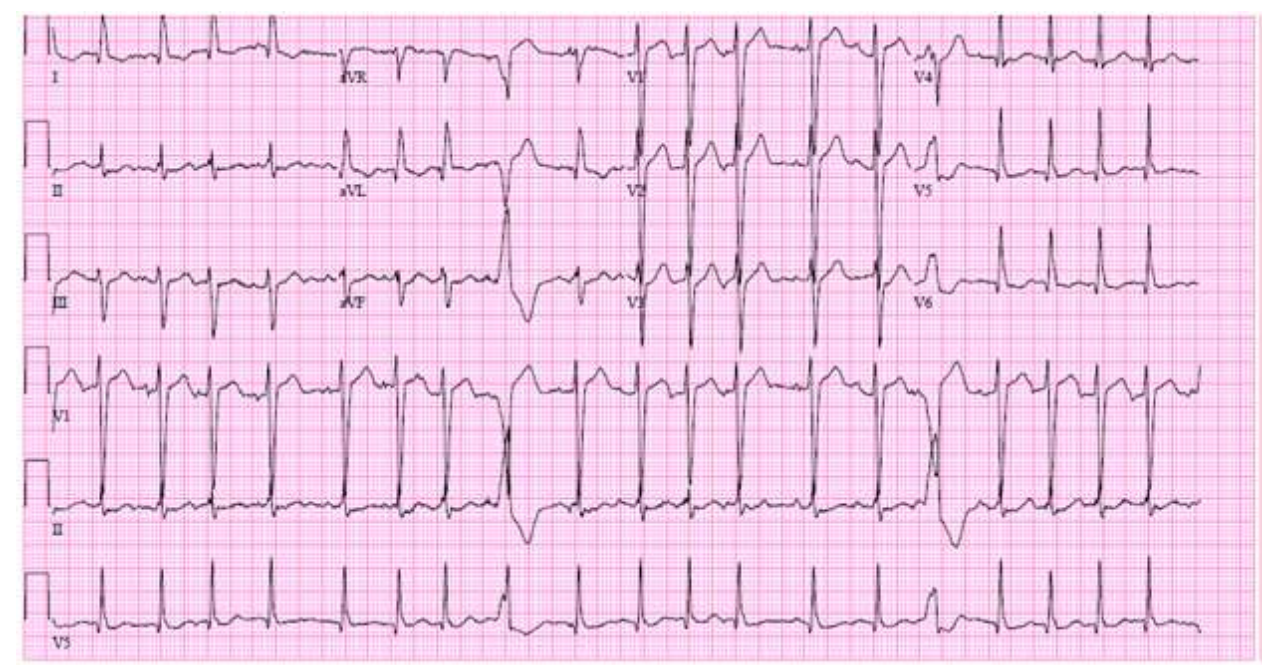

Figure 4: 12 Lead EKG showing Atrial Fibrillation with Rapid Ventricular Response

\section{References}

[1] D'CruzIA, Feghali N, Gross CM. Echocardiographic manifestations of mediastinal masses compressing or encroaching on the heart. Echocardiography 1994; 11:523-33.

[2] DeLuca A, Daniels S, Pathak N. Pulmonary edema due to extreme left atrial compression. $N \mathrm{~J} \mathrm{Med}$ 1991; 88:37-8.

[3] Chorro FJ, Cervantes A, Merino J, Solano C, Blanquer J, WasselA, et al. Extrinsic compression of the right atrium due to a mediastinal germ-cell tumor. Rev Esp Cardiol44: 553-555, 1991 (in Spanish)

[4] Van Besien K, Kelta M, Bahaguna P. Primary mediastinal B-cell lymphoma: a review of pathology and management. J ClinOncol. Mar 15 2001;19(6):1855-64

[5] Dubashi B, Cyriac S, Tenali SG. Clinicopathological analysis and outcome of primary mediastinal malignancies - A report of 91 cases from a single institute. Ann Thorac Med. Jul 2009;4(3):140-2

Volume 6 Issue 12, December 2017 www.ijsr.net 